Original article

\title{
Time-dependent effect of deuterium depletion on tumor growth and metastasis
}

\author{
Natalia V. Yaglova, Sergey S. Obernikhin, Valentin V. Yaglov, Svetlana V. Nazimova
}

Research Institute of Human Morphology, Moscow, Russia

Received 31 May 2019, Revised 5 November 2019, Accepted 27 April 2020

(C) 2019, Yaglova N.V., Obernikhin S.S., Yaglov V.V., Nazimova S.V.

(C) 2019, Russian Open Medical Journal

Abstract: Objective - impact of different time regimens of deuterium depletion on progression of syngeneic grafted tumor in mice was investigated.

Material and Methods - Experiment was performed on $64 \mathrm{C} 57 \mathrm{Bl} / 6$ mice divided into two groups with substitution of regular water for deuterium depleted water: 30 days prior to tumor cell inoculation and sinse the $1^{\text {st }}$ day of inoculation. Mice taking destilled mineralized water were considered as a control group. Half of the mice were injected melanoma B16 cells subcutaneously for assessment of survival rate and tumor growth inhibition index. The other mice were intravenously injected melanoma cells for estimation of lung hematogenous metastasis.

Results - Deuterium depletion began at the day of tumor inoculation did not change the parameters of survival rate ( $38.83 \pm 7.69$ days vs. $41.33 \pm 6.22$ days in the control). Tumor growth inhibition was found only at initial stage of tumor progression. Number of melanoma lung metastasis did not differ from the control values ( $32.30 \pm 6.12$ and $28.33 \pm 5.38$. consequently). The group of mice with preliminary consumption of deuterium depleted water demonstrated significantly higher survival rate $(70.10 \pm 16.20$ days), tumor inhibition index, and attenuation of lung metastasis $(6.0 \pm 1.20)$.

Conclusion - Our findings demonstrate that deuterium depletion exerts antitumor effect by both inhibition of tumor growth and metastasis, but development of the effect is time-dependent.

Keywords: deuterium, melanoma B16, deuterium-depleted water, survival rate, metastasis.

Cite as Yaglova NV, Obernikhin SS, Yaglov VV, Nazimova SV. Time-dependent effect of deuterium depletion on tumor growth and metastasis. Russian Open Medical Journal 2020; 9: e0210.

Correspondence to Natalia V. Yaglova. Address: Research Institute of Human Morphology, 3, Tsurupa st., Moscow, Russia. E-mail: yaglova@mail.ru.

\section{Introduction}

Neoplastic transformation is considered a complex of genetic, epigenetic, and metabolic changes directed to arrest transition of cells from proliferation to differentiation. Chemotherapy and radiotherapy, based on elimination of atypical cells, are conventional methods of anticancer treatment associated with multiple detrimental side effects. Drug delivery systems e.q. liposomes, niosomes, and nanoparticles, actively developed last two decades, have been considered a potent tool to enhance bioavailability and reduce side effects, but they also have limitations [1-3]. Targeted therapy based on suppression of specific genes or molecules contributing to malignant transformation and progression of tumor growth is a promising strategy to arrest cancer cells' division and spread. The main disadvantage of targeted therapy is ability to hit only one molecular target whereas a huge number of molecules and signaling pathways mediate malignant state of cells, and it also has serious side effects [4-6].

Novel scientific approaches to fight cancer require another targeting of malignant cells then discovery of specific tumor markers. Alteration of balance between levels of hydrogenium isotopes, namely protium and deuterium, in the mammalian organism have demonstrated unexpected biological effects which mechanisms are still poorly understood because of lack of knowledge on specific role of deuterium in the metabolism. Increasing evidence indicates that deuterium depletion in the organism exert positive effect on prevention of cancer progression. Recent studies have revealed that low level of deuterium promotes inhibition of cancer cell proliferation in vitro and in vivo $[7,8]$. Deuterium substitution for protium have prevented reparation of DNA and promoted apoptosis in cancer cells with not yet fully understood mechanisms of regulation of cell cycle $[7,9]$. These results are controversial because some studies revealed downregulation of proapoptotic genes expression in cancer cells after deuterium depletion [10].

Numerous studies demonstrate augmentation of cytotoxic effect of conventional antitumor chemotherapy after substitution of deuterium in the organism [9-11]. Today deuterium depleted water is suggested to be efficacious adjuvant to chemotherapy. It is well known that organisms are composed of up to $70 \%$ water. Therefore, consumption of deuterium depleted water is a main method of lowering deuterium concentration in the body. But antitumor effect of deuterium depletion is still only a demonstration of fact. The questions when the effect emerges and 
which degree of deuterium depletion produces the above mentioned effects are still without answer. Most in vivo experiments was performed on laboratory animals with xenografts of human cancer cell lines. Growth of xenogenic tumor requires preliminary suppression of immunity. Deprivation of natural antitumor defense impairs sanogenesis and complicates evaluation of deuterium depletion effects on cancer development and treatment.

In the present study we investigated impact of different time regimens of deuterium depletion on progression of syngeneic grafted tumor in mice.

\section{Material and Methods}

\section{Laboratory animals}

The experiment was performed on 64 male adult C57BI/ 6 mice (Scientific Center of Biomedical Technologies of Federal Medical and Biological Agency of Russia). The mice were kept in normal laboratory conditions at $22 \pm 2^{\circ} \mathrm{C}$ with equal periods of $12 \mathrm{~h}$ light and dark cycle and fed with standard chow diet ad libitum.

\section{Deuterium depletion regimens}

The mice were randomly distributed into 3 groups with different regimen of deuterium depletion. The first group $(n=22)$ received distilled mineralized deuterium depleted water (DDW) with 50ppm deuterium content (Kuban State University, Russia) ad libitum instead of tap water since the day of tumor inoculation (50 $\mathrm{ppm}$ [D]/0 group). The second group $(\mathrm{n}=20)$ began to drink deuterium depleted water (50 ppm) 30 days prior to inoculation (50 ppm [D]/-30 group). The third group ( $n=22)$, considered as control, received distilled mineralized water (146 ppm [D]) since the day of tumor inoculation.

\section{Subcutaneous tumor model}

The half of mice in each group were subcutaneously injected suspension of $5 \times 10^{5}$ murine melanoma B16 cells in $100 \mu$ of RPMI cell culture media into the right flank region. Murine melanoma cell line was obtained from Collection of tumor cell lines of Research Institute of Human Morphology. Viability of melanoma cells, assessed by MTT test, was about 95\%. 20 days after inoculation $100 \%$ of the mice exhibited visual and palpable tumor. Survival rate and average life span was assessed. Tumor sizes (longitudinal and cross diameters) were measured daily with calipers and tumor volume was calculated. Tumor growth inhibition index (TGI) was calculated as follows:

$\mathrm{TGI}=1-\frac{\text { mean volume of tumors in experimental group }}{\text { mean volume of tumors in the control }} \times 100 \%$

Autopsy and postmortem examination of inner organs was performed for every tumor-bearing animal.

\section{Lung metastasis model}

The other mice with different regimens of deuterium depletion were intravenously injected suspension of $10^{5}$ murine melanoma B16 cells in $100 \mu \mathrm{l}$ of RPMI cell culture media to produce hematogenous spread of melanoma cells [12]. 30 days after injection the mice were sacrificed by overdosage of halothane narcosis. Number of metastases in both lungs was estimated under stereomicroscope.

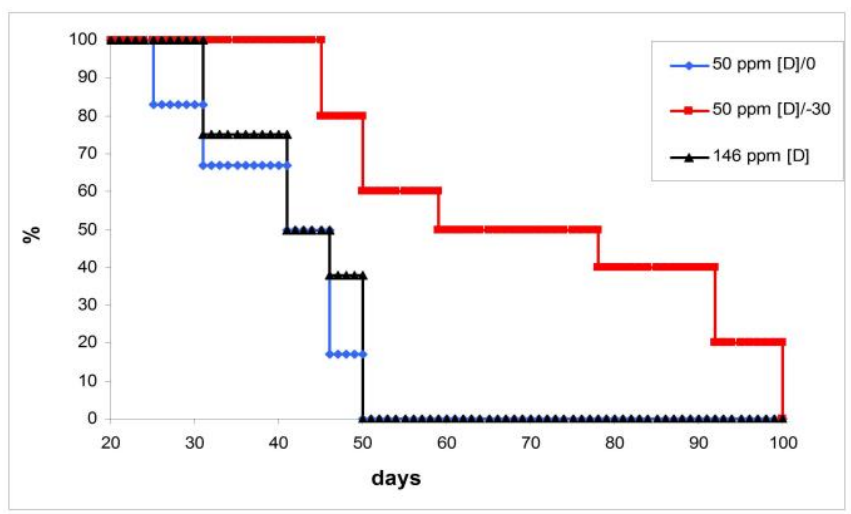

Figure 1. Survival analysis of melanoma-bearing mice with different time regimens of deuterium depletion.

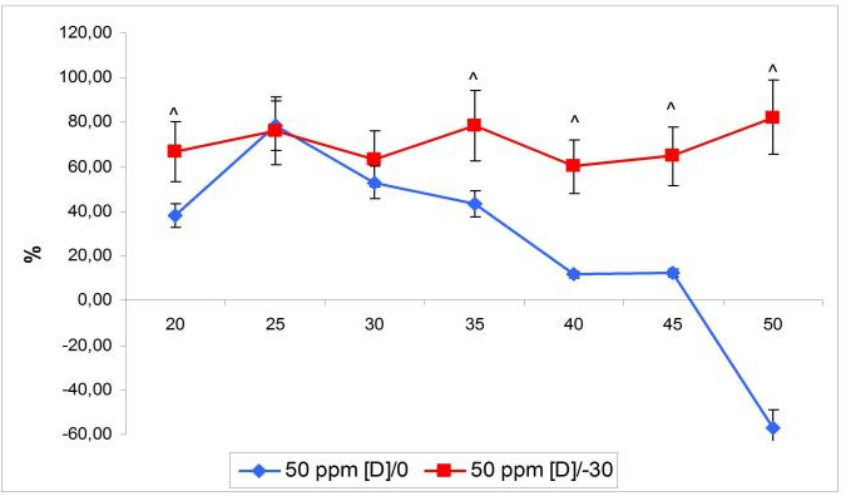

Figure 2. Tumor growth inhibition indices in melanoma-bearing mice with different time regimens of deuterium depletion $(\mathbf{M} \pm S D) . \wedge-p<0.05$ compared to group 50ppm [D]/0.

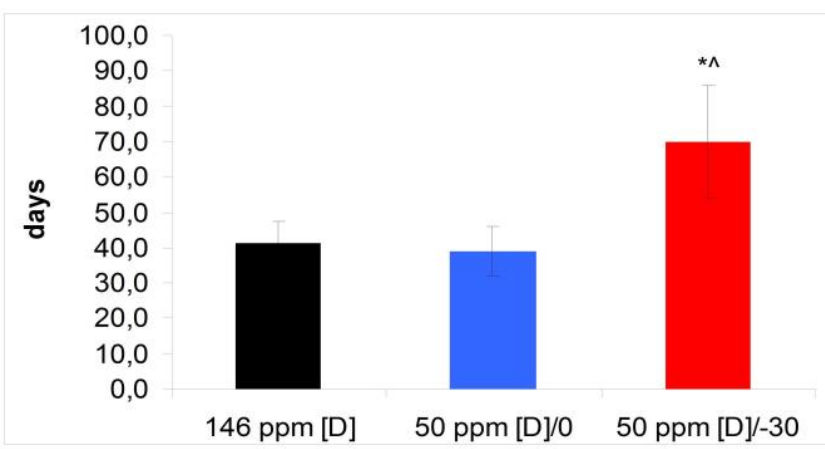

Figure 3. Number of lung metastasis in mice with different time regimens of deuterium depletion after intravenous injection of melanoma B16 cells (M $\pm S D){ }^{*}-p<0.05$ compared to group $146 p p m[D], \wedge-p<0.05$ compared to group 50ppm [D]/0.

\section{Statistical analysis}

The statistical analyses were carried out using the software package Statistica 7.0 (StatSoft, USA). The log-rang test was used for comparison of survival curves. The central tendency and dispersion of traits with approximately normal distribution were presented as the mean and standard error of the mean $(\mathrm{M} \pm \mathrm{m})$. Quantitative comparisons of independent groups were performed using Student's t test taking into account the values of Levene's test for the equality of variances and $\chi^{2}$. Differences were considered statistically significant at $p<0.05$. 
Table 1. Lifespan parameters of tumor-bearing mice with different regimens of deuterium depletion

\begin{tabular}{|c|c|c|c|}
\hline \multirow{2}{*}{ Parameter } & \multicolumn{3}{|c|}{ Group } \\
\hline & 146 ppm [D] (control) & $50 \mathrm{ppm}[\mathrm{D}] / 0$ & $50 \mathrm{ppm}[\mathrm{D}] /-30$ \\
\hline Minimal lifespan & 31 & 25 & 45 \\
\hline Maximal lifespan & 50 & 50 & 100 \\
\hline
\end{tabular}

Data are presented as the mean and standard deviation $-M \pm S D$. Significant differences $(p<0.05)$ in comparison with: * - control, $\wedge-50$ ppm $[D] / 0$ group.

\section{Results}

\section{Survival analysis}

The survival distribution of the control animals (146 ppm [D]) revealed gradient changes of survival rate from $25^{\text {th }}$ to $50^{\text {th }}$ day (Figure 1). The survival rate of mice receiving DDW since tumor inoculation was similar to the control group and the average lifespan, therefore, did not differ from the control (Figure 1, Table 1). The mice with preliminary deuterium depletion demonstrated statistically significant increase of survival rate with gradient death of tumor-bearing mice (Figure 1). Their minimal and maximal lifespans were significantly longer than in the previous groups (Table 1). The average lifespan exceeded the control values by 1.7 times, the values of mice treated with DDW since tumor inoculation by 1.8 times (Table 1). Autopsy of the control and DDW-receiving mice revealed multiple melanoma metastases, mainly in the lungs, liver and spleen.

\section{Tumor growth inhibition}

Inhibition of tumor growth in the group with deuterium depletion since tumor inoculation demonstrated significant raise from $20^{\text {th }}$ to $25^{\text {th }}$ day with subsequent gradual decrease. From $45^{\text {th }}$ to $50^{\text {th }}$ day tumor growth was more intensive than in the control group (Figure 2). The mice which started to consume DDW 30 day prior to tumor inoculation exhibited higher inhibition of tumor growth. Their tumor growth inhibition index did not significantly change from $20^{\text {th }}$ to $45^{\text {th }}$ day and tended to increase from $45^{\text {th }}$ to $50^{\text {th }}$ day (Figure 2).

\section{Metastasis}

Visual examination did not find any differences in number and location of lung metastases in the control group and receiving DDW since inoculation group (Figure 3 ). Preliminary administration of DDW resulted in striking decrease of melanoma lung metastases (Figure 3).

\section{Discussion}

B16 melanoma, a murine tumor cell line, is frequently used for study of solid tumor formation due to high malignant potential. Melanoma cells may begin to spread even at early stages of tumor development that is why it is also used for metastasis modeling $[12,13]$. The fact that every mouse developed solid tumor after subcutaneous inoculation indicates the proper dosage of tumor cells injected. The mice within the control group showed similar rate of tumor formation after inoculation of melanoma cells. The survival analysis revealed that $50 \%$ of the control mice died on the $41^{\text {st }}$ day after tumor cell injection, and the average lifespan was also 41 day. It proves that tumor progress and metastasis in the control animals had the same rate. Numerous metastases found in postmortem examination of mice are considered a primary cause of death. Deuterium depletion which began from the moment of tumor grafting did no change parameters of survival rate in the mice exept minimal lifespan. Survival rate of $50 \%$ of DDW-treated animals and their average lifespan were also similar. Longer minimal life span and significant inhibition of tumor growth during 15 days after solid tumor formation indicate that deuterium depletion somehow prevented rapid tumor growth at initial stage. It corresponds with data of S. Paryad-zanjani et al., that DDW consumption decreases melanoma F10 growth rate in C57BI/6 mice within 30 days after grafting [14]. Our data demonstrate that antitumor effect of deuterium depletion gradually reduces after $25^{\text {th }}$ day since tumor inoculation. One month earlier replacement of tap water with DDW significantly increased all parameters of survival rate. Longer lifespan and higher tumor growth inhibition indices prove that lowered deuterium content by the moment of tumor cell inoculation inhibited both tumor growth and metastasis. Stable suppression of melanoma growth within 50 day after grafting might be mediated by inhibition of proliferation or activation of apoptosis of tumor cells $[8,15]$. Perhaps, both mechanisms were involved. Replacement of regular water to DDW with deuterium content less than $50 \mathrm{ppm}$ during one month is known to significantly reduce deuterium concentration in the organism [16], and increase of duration of DDW consumption enhance its physiological effects [17]. So, differences in tumor growth inhibition indices in mice with different regimens of DDW consumption demonstrate time-depending effect of deuterium depletion.

Assessment of lung metastases after intravenous injection of melanoma cells demonstrates that preliminary depletion of deuterium content in the organism attenuates spread of tumor cells. Inhibition of metastasis, therefore, facilitated elongation of the lifespan. Deuterium depletion, began simultaneously with tumor process, did not inhibit hematogenous spread of tumor cells. Poorly studied role of deuterium atoms in metabolism, cell division and growth do not allow to establish mechanisms of antitumor effect. It is very important to note, that deuterium depletion did not prevent formation of tumor after inoculation of melanoma cells and only suppressed tumor growth and metastasis. Attenuated metastasis may be explained by changes produced in extracellular matrix and adjusted blood and lymphatic vessels. Malignant cells are known to secrete proteases which digest extracellular matrix and facilitate penetration into lymphatic and blood vessels $[18,19]$. Extravazation of tumor cells also requires penetration through vessel wall. Low metastasis rate suggests that deuterium depletion strengthens extracellular matrix and blood vessel wall and makes connective tissues more stable to degradation by tumor enzymes. But this hypothesis is not in convenience with data on nature of deuterium chemical bonds with biogenic atoms. It has been shown that carbon bond with deuterium is stronger than with protium and requires more energy for dissociation [20]. It means that deuterium depletion reduces number of stronger C-D bonds in proteins making the latter less stable for enzymatic degradation. Results of several studies show that deuterium depletion upregulates immunity [21]. Therefore, antitumor effect also might be facilitated by activation of 
antitumor immune response. The fact that even preliminary deuterium depletion failed to prevent formation of tumor does not allow to reject the hypothesis of immune system implication because of huge number of injected syngeneic tumor cells. The data obtained show ambiguous effect of deuterium depletion on tumor progression and identify novel areas of research on mechanisms of deuterium effects.

\section{Conclusion}

In conclusion, our findings demonstrate that deuterium depletion exerts antitumor effect by inhibiting both tumor growth and metastasis. But development of antitumor effect requires preliminary depletion of deuterium content. It indicates that antitumor effect depends not only on degree of deuterium depletion. A complex of physiological changes, produced by deuterium depletion, is probably also mediated attenuation of tumor growth. Alteration of physiological parameters requires certain period of time to develop that is why only preliminary deuterium depletion demonstrated antitumor effect.

\section{Ethical approval}

The experiment was approved by local Ethics committee and performed according to guidance «International guiding principles for biomedical research involving animals».

\section{Conflict of interest}

The authors declare that they have no conflict of interest.

\section{Aknowledgements}

The reported study was partially funded by RFBR, project number 20015-00236 A

\section{References}

1. Berezov TT, laglova NV, Dmitrieva TB, Zhirkov luA, Chekhonin VP. Liposome-oriented transport of therapeutic drugs. Vestn Ross Akad Med Nauk 2004; (5): 42-47. Russian. https://pubmed.ncbi.nlm.nih.gov/15320549.

2. Fedoseeva VV, Khalansky AS, Mkhitarov VA., Tsvetkov IS, Malinovskaya YA, Maksimenko OO, et al. Anti-tumor activity of doxorubicin-loaded poly(lactide-co-glycolide) nanoparticles in the experimental glioblastoma. Clin Exp Morphol 2017; (2): 65-71. https://elibrary.ru/item.asp?id=29946269.

3. Rajera R, Nagpal K, Singh SK, Mishra DN. Niosomes: a controlled and novel drug delivery system. Biol Pharm Bull 2011; 34(7): 945-953. https://doi.org/10.1248/bpb.34.945.

4. Chang ST, Menias CO, Lubner MG, Mellnick VM, Hara AK, Desser TS. Molecular and Clinical Approach to Intra-abdominal Adverse Effects of Targeted Cancer Therapies. Radiographics 2017; 37(5): 1461-1482. https://doi.org/10.1148/rg.2017160162.

5. Macdonald JB, Macdonald B, Golitz LE, LoRusso P, Sekulic A. Cutaneous adverse effects of targeted therapies: Part II: Inhibitors of intracellular molecular signaling pathways. J Am Acad Dermatol 2015; 72(2): 221-236; quiz 237-238. https://doi.org/10.1016/j.jaad.2014.07.033.

6. Kamimura N, Wolf AM, Iwai Y. Development of Cancer Immunotherapy Targeting the PD-1 Pathway. J Nippon Med Sch 2019; 86(1): 10-14. https://doi.org/10.1272/jnms.jnms.2019_86-2.

7. Yavari K, Kooshesh L. Deuterium depleted water inhibits the proliferation of human MCF7 breast cancer cell lines by inducing cell cycle arrest. Nutr Cancer 2019; 71(6): 1019-1029. https://doi.org/10.1080/01635581.2019.1595048.
8. Cong FS, Zhang YR, Sheng HC, Ao ZH, Zhang SY, Wang JY. Deuteriumdepleted water inhibits human lung carcinoma cell growth by apoptosis. Exp Ther Med 2010; 1(2): 277-283. https://doi.org/10.3892/etm_00000043.

9. Hassanzade A, Mandegary A, Sharif E, Rasooli R, Mohammadnejad R, Masoumi-Ardekani $Y$. Cyclooxygenase inhibitors combined with deuterium-enriched water augment cytotoxicity in A549 lung cancer cell line via activation of apoptosis and MAPK pathways. Iran J Basic Med Sci 2018; 21(5): 508-516. https://doi.org/10.22038/ijbms.2018.25366.6269.

10. Gyongyi Z, Budan F, Szabo I, Ember I, Kiss I, Krempels K, et al. Deuterium depleted water effects on survival of lung cancer patients and expression of Kras, Bcl2, and Myc genes in mouse lung. Nutr Cancer 2013; 65(2): 240-246. https://doi.org/10.1080/01635581.2013.756533.

11. Krempels K, Somlyai I, Gyöngyi Z, Ember I, Balog K, Abonyi O, et al. A retrospective study of survival in breast cancer patients undergoing deuterium depletion in addition to conventional therapies. J Cancer Res Ther 2013; 1(8): 194-200. http://doi.org/10.14312/2052 4994.2013-29.

12. Giavazzi R, Decio A. Syngeneic murine metastasis models: $B 16$ melanoma. Methods Mol Biol 2014; 1070: 131-140. https://doi.org/10.1007/978-1-4614-8244-4_10.

13. Walker GJ, Soyer HP, Terzian T, Box NF. Modelling melanoma in mice. Pigment Cell Melanoma Res 2011; 24(6): 1158-1176. https://doi.org/10.1111/j.1755-148x.2011.00923.x.

14. Paryad-zanjan S, Mahjoob MJ, Amanpour S, Kheirbakhsh R, Haji Akhoundzadeh M. A supplemental treatment for chemotherapy: control simulation using a mathematical model with estimated parameters based on in vivo experiment. IFAC-PapersOnLine 2016; 49(26): 277-282. https://doi.org/10.1016/j.ifacol.2016.12.138.

15. Laskay G, Somlyai G, Jakli G. Effects of deuterium depletion on proliferation and apoptosis in cultured murine haemopoietic cells. Eur Chem Bull 2012; 1(1):44. https://doi.org/10.17628/ecb.2012.1.39-47.

16. Artsybasheva O, Barysheva E, Shashkov D, Vlasov R, Tekutskaya E. Changes of oxidation during use the food diet with deuterium depleted water in laboratory animals with purulent inflammation. Russ Open Med J 2014; 3: 0201. https://doi.org/10.15275/rusomj.2014.0201.

17. Lisitsyn AB, Baryshev MG, Basov AA, Barysheva EV, Bykov IM, Dydykin $A S$, et al. Influence of deuterium depleted water on the organism of laboratory animals in various functional conditions of nonspecific protective systems. Biofizika 2014; 59(4): 757-765. Russian. https://pubmed.ncbi.nlm.nih.gov/25707244.

18. Damsky WE, Theodosakis N, Bosenberg M. Melanoma metastasis: new concepts and evolving paradigms. Oncogene 2014; 33(19): 2413-2422. https://doi.org/10.1038/onc.2013.194.

19. Redmer T. Deciphering mechanisms of brain metastasis in melanoma the gist of the matter. Mol Cancer 2018; 17(1): 106. https://doi.org/10.1186/s12943-018-0854-5.

20. Enkelmann DD, Hofmann DWM, Merz K. Deuterium Shifts the Equilibrium: How Heavy Water Can Influence Organic Multicomponent Crystal Formation. Cryst Growth Des 2017; 17(9): 4726-4729. https://doi.org/10.1021/acs.cgd.7b00654.

21. Bild W, Stefanescu I, Haulica I, Lupuşoru C, Titescu G, lliescu R, et al. Research concerning the radioprotective and immunostimulating effects of deuterium-depleted water. Rom J Physiol 1999; 36(3-4): 205218. https://pubmed.ncbi.nlm.nih.gov/11797936.

\section{Authors:}

Natalia V. Yaglova - MD, DSc, Head of the Laboratory of Endocrine System Development, Research Institute of Human Morphology, Moscow, Russia. http://orcid.org/0000-0002-8239-5039.

Sergey S. Obernikhin - MD, DSc, Senior Researcher, Laboratory of Endocrine System Development, Research Institute of Human Morphology, Moscow, Russia. http://orcid.org/0000-0003-0997-6766.

Valentin V. Yaglov - MD, DSc, Professor, Chief Researcher, Laboratory of Endocrine System Development, Research Institute of Human Morphology, Moscow, Russia. http://orcid.org/0000-0003-4357-0639. 
Svetlana V. Nazimova - MD, PhD, Senior Researcher, Laboratory of Endocrine System Development, Research Institute of Human Morphology, Moscow, Russia. http://orcid.org/0000-0001-5118-7037. 